\title{
Pankreaskarzinom: Kriterien und Grenzen der Resektabilität
}

\author{
Helmut Witzigmann Henry Jungnickel Stefan Kißenkötter \\ Klinik für Allgemein- und Viszeralchirurgie, Krankenhaus Dresden-Friedrichstadt - Akademisches Lehrkrankenhaus der TU Dresden, \\ Deutschland
}

\section{Schlüsselwörter \\ Pankreaskarzinom · Resektabilität . \\ Lymphknotenmetastasen · Gefäßinvasion}

\section{Zusammenfassung}

Ziel einer Definition der Resektabilität von Pankreaskarzinomen ist die Beschreibung von Kriterien, welche eine potentielle R0-Resektion ermöglichen. Wenngleich es zur Frage der Resektion bei positiven regionären Lymphknoten keine kontrollierten Studien gibt, ist weltweit die Resektion bei regionären Lymphknotenmetastasen Standard. Positive interaortokavale Lymphknoten stellen trotz Klassifikation als Fernmetastasen (M1) keine absolute Kontraindikation zur Resektion dar. Die mesenterikoportale Venenresektion bei Verdacht auf Tumorinfiltration ist ein sicheres Verfahren. Sie hat keinen negativen Einfluss auf Morbidität, Mortalität und Überleben. Die En-bloc-Resektion der Arteria hepatica und der Arteria mesenterica superior wird sehr kontrovers diskutiert und sollte nur in Einzelfällen erwogen werden. Bei den meist fortgeschrittenen Karzinomen des Pankreaskorpus und -schwanzes kann durch eine En-bloc-Resektion des Truncus coeliacus eine höhere R0-Resektionsrate erreicht werden.

\section{Einleitung}

Die Prognose eines Patienten mit duktalem Pankreaskarzinom wird - wie bei anderen Malignomen - in erster Linie von der R0-Resektabilität bestimmt [1]. Die Karzinome des Pankreas führen frühzeitig zur Invasion des peripankreatischen

\section{Keywords}

Pancreatic cancer · Resectability .

Lymph node metastasis · Vascular invasion

\section{Summary}

Pancreatic Cancer: Criteria and Limits of Resectability The aim of defining the resectability of pancreatic cancer is to determine the indication for potential $\mathrm{R} 0$ resection. Despite the absence of controlled trials, tumor resection in patients with regional lymph node involvement is a standard procedure worldwide. The involvement of interaortocaval lymph nodes is not an absolute contraindication for resection, although they are classified as distant metastasis (M1). Major pancreatic surgery can be safely combined with en-bloc resection of mesenteric, portal and splenic veins. Postoperative morbidity and mortality and long-term survival in patients with vein resection are comparable with those of patients without vein resection. The role of arterial en-bloc resection of the hepatic artery and the superior mesenteric artery is highly controversial and should be considered only in selected patients. For patients with locally advanced cancer of the body and tail of the pancreas distal pancreatectomy with en-bloc celiac axis resection offers an improved $\mathrm{R} 0$ resection rate.

\section{KARGER}

Fax +497614520714

Information@Karger.de

www.karger.com (c) 2010 S. Karger GmbH, Freiburg

Accessible online at:

www.karger.com/vim
Gewebes und zur perineuralen Infiltration. Des Weiteren kommt es zur frühzeitigen Invasion der Adventitia der großen Gefäße, vor allem der Vena mesenterica superior und der Pfortader. Die chirurgische Therapie des Pankreaskarzinoms ist durch eine Zunahme der Resektabilitätsrate und eine Abnahme der Mortalität und Morbidität gekennzeichnet. 
Zur Fragestellung der grundsätzlichen Selektion von Patienten für eine Resektion, der Operation bei Lymphknotenmetastasen, der Gefäßresektion bei Verdacht auf eine arterielle/venöse Gefäßinfiltration und der palliativen und Rezidivresektion gibt es wenige kontrollierte Daten. Bei der Einschätzung der Resektabilität anhand der präoperativen Bildgebung und des intraoperativen Situs spielt die Erfahrung des Chirurgen eine zentrale Rolle. Der intraoperative Überraschungsbefund «Irresektabilität» liegt heutzutage aufgrund der verbesserten präoperativen Bildgebung unter $10 \%$.

Wenn die Kriterien und Grenzen der Resektabilität definiert werden, müssen die Aspekte der onkologischen Sinnhaftigkeit und der technischen Machbarkeit berücksichtigt werden. Die funktionellen Folgen eines pankreatopriven Diabetes mellitus und der exokrinen Pankreasinsuffizienz sind heutzutage keine Kontraindikationen für eine Resektion. Selbst nach totaler Pankreatektomie sind schwere metabolische endokrine Komplikationen durch den Einsatz neuer Verzögerungsinsuline und bei guter Betreuung durch einen Diabetologen selten geworden.

In der aktuellen S3-Leitlinie zur Diagnostik und Therapie des exokrinen Pankreaskarzinoms wurden folgende Kriterien der Irresektabilität von Seiten des Patienten und des Tumors definiert [2]:

- Infiltration des Truncus coeliacus und der Arteria mesenterica superior (Empfehlungsgrad: C, Evidenzstärke 4, starker Konsens),

- Fernmetastasen (Empfehlungsgrad: A, Evidenzstärke 3, Konsens),

- Komorbidität kann ein Kriterium sein, bei einem Patienten auf eine Resektion zu verzichten (Empfehlungsgrad: C, Evidenzstärke 4, starker Konsens).

In diesem Artikel werden folgende Fragen der Resektabilität von Pankreaskarzinomen diskutiert:

- Resektion bei Lymphknotenbefall,

- Resektion bei Verdacht auf venöse/arterielle Infiltration,

- Indikation zur Resektion bei fortgeschrittenen Karzinomen des Pankreaskorpus und -schwanzes.

Die Fragen der Resektion von Lebermetastasen und Lokalrezidiven werden von anderen Autoren in diesem Heft behandelt.

\section{Resektion bei Lymphknotenbefall}

Der Lymphknotenstatus ist beim Pankreaskarzinom einer der wichtigsten Prognoseparameter. Bei Nachweis von Lymphknotenmetastasen liegt die 5-Jahres-Überlebensrate bei zirka $10 \%$, bei metastasennegativem Lymphknotenstatus hingegen bei 25-30\% [3]. Die Definition einer extendierten Lymphknotendissektion ist uneinheitlich. In einer internationalen Konsensuskonferenz wurde eine Unterscheidung in Standardpankreatoduodenektomie, radikale Pankreatoduodenektomie und ausgedehnte radikale Pankreatoduodenek- tomie vorgeschlagen [4]. Die aktuelle S3-Leitlinie «Exokrines Pankreaskarzinom» definiert die standardisierte Lymphadenektomie beim Pankreaskopfkarzinom wie folgt: Komplette und zirkuläre Ausdissektion der Lymphknoten des Ligamentum hepatoduodenale, der Lymphknoten im Bereich der Arteria hepatica communis, der Pfortader, des kranialen Anteils der V. mesenterica superior, des Truncuscoeliacus-Lymphknoten rechts und der rechten Hemizirkumferenz des Stammes der A. mesenterica superior [2]. Eine Meta-Analyse zu mehreren prospektiv randomisierten Studien [5-8] durch Michalski et al. [9] erbrachte keinen Überlebensvorteil durch eine extendierte Lymphknotendissektion. Sie zeigte hingegen einen Trend für eine erhöhte Morbidität. In der aktuellen S3-Leitlinie «Exokrines Pankreaskarzinom» wird daher geschlussfolgert: «Ein Benefit der tatsächlich erweiterten Resektion konnte bisher nicht nachgewiesen werden. Ziel einer jeden Resektion muss die R0Resektion sein» (Empfehlungsgrad: B, Evidenzstärke 2b, Konsens) [2].

In der aktuellen TNM-Klassifikation der UICC von 2010 werden Metastasen in regionären Lymphknoten als N1 versus keine Lymphknotenmetastasen $=$ N0 unterschieden [10]. Die regionären Lymphknoten werden in superiore, inferiore, anteriore, posteriore, lienale und zöliakale Lymphknoten unterteilt. Paraaortale Lymphknotenmetastasen gelten als Fernmetastasen (M1). In der Klassifikation der Lymphknotenstationen der Japan Pancreas Society (JPS) werden die intraabdominellen Lymphknotenstationen durchnummeriert und in drei Gruppen klassifiziert (JPS N1-3) [11]. Lymphknotenmetastasen der Gruppe 3 (JPS N3) werden als Fernmetastasen (M1) gewertet. Hierunter fallen beim Pankreaskopfkarzinom Lymphknoten links und rechts der Kardia, an kleiner und großer Kurvatur des Magens und suprapylorisch, entlang des unteren Rands von Pankreaskorpus und -schwanz, am Milzhilus, entlang der Arteria gastrica sinistra, der Arteria lienalis, der Arteria colica media, am Truncus coeliacus, und entlang der Aorta vom oberen Rand des Truncus coelicacus bis zum oberen Rand der A. mesenterica inferior. Beim Karzinom im Pankreaskorpus und -schwanzbereich zählen zur Gruppe 3 (JPS N3) die Lymphknoten entlang der Arteria hepatica (propria), an der Vena portae, entlang des Ductus choledochus, supra- und infrapylorisch, an der Vorder- und Rückseite des Pankreaskopfs und ebenfalls entlang der Aorta vom oberen Rand des Truncus coelicacus bis zum oberen Rand der Arteria mesenterica inferior.

Die präoperative Einschätzung des Lymphknotenstatus anhand der Bildgebung ist unzuverlässig. Kim et al. [12] zufolge kann weder mittels CT noch MRT eine zuverlässige Aussage zur Metastasierung in paraaortale Lymphknoten gemacht werden (Sensitivität 50 bzw. 77,8\%, Spezifität 91,1 bzw. $66,4 \%$ bei einer Lymphknotenlänge über 11,6 mm bzw. Lymphknotenbreite über $5,3 \mathrm{~mm}$ ). Lediglich bei einer zentralen Nekrose liegt der positiv prädiktive Wert bei nahezu $100 \%$. In Bezug auf paraaortale Lymphknotenmetastasen 
weist die PET im Vergleich zur CT eine bessere Spezifität (100 vs. $84,9 \%$ ), aber eine schlechtere Sensitivität (46,2 vs. 61,5\%) auf [13]. Die besten Ergebnisse zur Detektion einer Lymphknotenmetastasierung beim Pankreaskarzinom liefert die Endosonographie [14, 15].

Reaktiv vergrößerte Lymphknoten können fälschlicherweise als Metastasen angesehen und den Patienten damit die Chance auf eine Tumorresektion genommen werden [12, 16, 17]. Den Ergebnissen mehrerer Studien zufolge ist jedoch weniger die Unterscheidung N+ versus N0 prognostisch entscheidend, sondern der Quotient aus der Anzahl positiver Lymphknoten geteilt durch die Anzahl der untersuchten Lymphknoten (LK-Ratio) [18-22]. In einer Studie von Riediger et al. [21] ergab die klassische Einteilung in N0 und N1 keinen statistisch signifikanten Einfluss auf das Überleben. Dagegen erwies sich eine LK-Ratio $\geq 0,3$ als wichtigster prognostischer Faktor (RR 2,2). Die prognostische Relevanz lag damit höher als beim Resektionsstatus R+ versus R0 (RR 1,7) und dem Differenzierungsgrad des Tumors (RR 1,5).

In der Arbeit von Connor et al. [23] erwiesen sich positive Lymphknoten an der A. hepatica communis (JPS Lymphknoten(LK)8a) als ein unabhängiger negativer Prognosefaktor [23]. Diese Station entspricht JPS N2 wenn der Primärtumor im Pankreaskopf liegt bzw. JPS N1 bei Lokalisation des Primärtumors in Pankreaskorpus/-schwanz. Das mediane Überleben sank bei positivem JPS LK8a signifikant auf 199 versus 417 Tage ohne Lymphknotenmetastasen im Bereich der A. hepatica communis. Zusätzlich wurden in dieser Studie die Lymphknoten paraaortal zwischen dem Unterrand der Vena renalis sinistra bis zum Oberrand der A. mesenterica inferior untersucht (JPS LK16b1). Sie werden in der japanischen Klassifikation, unabhängig von der Lokalisation des Primärtumors im Pankreas, der Gruppe JPS N3 zugeordnet und gelten damit, der TNM-Klassifikation entsprechend, bei Tumorbefall als Fernmetastasen (M1). Im Gegensatz zur oben genannten signifikanten Prognoseverschlechterung bei Lymphknotenbeteiligung an der A. hepatica communis hatte eine Metastasierung der JPS LK16b1 keinen negativen Effekt auf die Prognose (medianes Überleben 457 vs. 503 Tage). Es fand sich jedoch bei einer Lymphknotenmetastasierung paraaortal signifikant häufiger eine R1-Resektion des posterioren Absetzungsrands. Das mediane Überleben ohne Resektion lag in dieser Studie bei 98 Tagen.

Auch Kayara et al. [24] konnten in ihrer Studie keinen Unterschied im Überleben bei Patienten mit regionären Lymphknotenmetastasen mit versus ohne zusätzliche Beteiligung paraaortaler Lymphknoten nachweisen.

Eine Arbeitsgruppe an der University of Nagoya, Japan, untersuchte anhand einer prospektiven Datenbank den Einfluss der unterschiedlichen Lymphknotenstationen der japanischen Klassifikation auf das Überleben. In einer Analyse der operierten Patienten zwischen 1981 und 2005 bzw. 2007 zeigte sich zwar eine signifikant schlechtere Prognose bei JPS N2/N3 versus N0-Status, jedoch konnte kein signifikanter Unter- schied zwischen den Untergruppen JPS N1, N2 und N3 gezeigt werden [25]. In einer Subanalyse war das durchschnittliche Überleben bei einer Metastasierung paraaortal (JPS LK16+) vergleichbar zu dem der Patienten, bei denen keine Resektion erfolgte und widerspricht damit den Ergebnissen von Connor et al. [23] und Kayara et al. [24]. Jedoch gab es auch in dieser Studie Langzeitüberlebende mit JPS-LK16positivem Befund. Eine multivariate Analyse identifizierte als unabhängige, prognostisch günstige Faktoren ein Alter über 60 Jahre, eine Tumorgröße kleiner $4 \mathrm{~cm}$ und die fehlende Pfortaderinfiltration. Die Arbeitsgruppe der University of Nagoya schlussfolgerte daher, dass die Patienten, bei denen diese Kriterien zutreffen, auch bei paraaortalen Lymphknotenmetastasen von einer Resektion profitieren können [26]. Bei extendierter Lymphknotendissektion fanden Sakai et al. [27] bei $19 \%$ der Patienten Metastasen in paraaortalen Lymphknoten.

\section{Konklusion}

Wenngleich es zur Frage der Resektion bei regionären Lymphknotenmetastasen keine kontrollierten Studien gibt, ist weltweit die Resektion bei positiven regionären Lymphknoten Standard. Positive interaortokavale Lymphknoten stellen trotz Klassifikation als Fernmetastasen TNM: M1 bzw. JPS: N3/M1) keine absolute Kontraindikation zur Resektion dar. Bei positiven interaortokavalen Lymphknoten sollten Patientenalter, Tumorgröße und Gefäßinfiltration bei der Indikationsstellung zur Operation berücksichtigt werden.

\section{Resektion bei Verdacht auf Gefäßinfiltration}

Eine Unterscheidung zwischen Tumorinfiltration und Entzündungsadhäsion ist sowohl in der präoperativen Bildgebung als auch intraoperativ sehr schwierig. Das Ziel einer En-bloc-Resektion des Tumors mit Gefäßen bei Verdacht auf Gefäßinfiltration ist das Erreichen einer potentiellen R0Resektion. Ein therapeutischer Nihilismus ist bei Befall der $\mathrm{V}$. portae, V. mesenterica superior und V. lienalis aus technischen und prognostischen Gründen heutzutage nicht mehr vertretbar. Sehr kontrovers diskutiert wird die Gefäßresektion bei Befall der A. hepatica, des Truncus coeliacus und der A. mesenterica superior.

Bei der Diskussion der Grenzen der Resektabilität in Bezug auf die Gefäße müssen folgende Fragen beantwortet werden:

- Ist die perioperative Morbidität und Mortalität bei Mitresektion von Gefäßen höher?

- Ist die onkologische Prognose bei Vorliegen einer Gefäßinfiltration schlechter?

In der Studie von Yekebas et al. [28] zeigten sich bei 136 Patienten mit Gefäßresektion ( $\mathrm{n}=128$ mesenterikoportal; $\mathrm{n}=8$ A. hepatica oder A. mesenterica superior; $n=5$ arterielle und venöse Resektion) eine vergleichbare Morbidität und Morta- 
lität (40,4 und 3,7\%) im Vergleich zu Pankreasresektionen ohne Mitresektion von Gefäßen (39,7 und 4,0\%) [28].

Die En-bloc-Resektion der Mesenterialvene bzw. Pfortader stellt in Zentren inzwischen bei fortgeschrittenen Tumoren des Pankreaskopfs ein gängiges Verfahren zur Erzielung von R0-Resektionen dar. Riediger et al. [29] zeigten in einer Studie an 222 Patienten mit Pankreaskopfresektion eine vergleichbare perioperative Morbidität und Mortalität nach Resektionen mit und ohne mesenterikoportale Venenresektion. Die histologische Untersuchung ergab bei $60 \%$ eine «echte Gefäßinfiltration». Auch das Langzeitüberleben unterschied sich nicht zwischen den Patienten mit und ohne mesenterikoportale Venenresektion [29]. In Übereinstimmung mit dieser Studie fand auch die Arbeitsgruppe von Kaneoka et al. [30] keinen Unterschied im 5-Jahres-Überleben von Patienten mit versus ohne mesenterikoportale Venenresektion (23 vs. 34\%). Allerdings betrug das 5-Jahres-Überleben in der Subgruppe von Patienten mit einem Venenbefall über eine Strecke von mehr als $3 \mathrm{~cm}$ nur 4\% [30]. Die Mitresektion und Rekonstruktion von arteriellen Gefäßen ergab in einer Fallserie von $23 \mathrm{~Pa}$ tienten von Amano et al. [31] einen Überlebensvorteil, wenn eine R0-Resektion erreicht wurde. In der Studie von Yekebas et al. [28] verstarb 1 Patient perioperativ, und von 13 Patienten mit Mitresektion der A. hepatica / A. mesenterica superior lebten 4 Patienten länger als 2 Jahre. Drei dieser 4 Patienten hatten eine histologisch nachgewiesene Gefäßinfiltration, und bei allen 4 Patienten fand sich ein pN0-Tumorstadium. In einer Arbeit von Nakao et al. [25] betrug die OP-Mortalität nach mesenterikoportaler Enbloc-Resektion 2,7\% und nach Mitresektion von Arterien $35,7 \%$ [25]. Settmacher et al. [32] publizierten eine Serie von 10 Patienten, bei denen im Rahmen einer Pankreasresektion eine Viszeralarterienrekonstruktion mit Homografts erfolgte. Vorteil der arteriellen Homografts ist ihre Resistenz gegenüber lokalen Infektionen.

\section{Konklusion}

Die mesenterikoportale Venensektion bei Verdacht auf Tumorinfiltration ist ein sicheres Verfahren. Sie hat keinen negativen Einfluss auf Morbidität, Mortalität und Überleben. Die En-bloc-Resektion der A. hepatica und der A. mesenterica superior wird sehr kontrovers diskutiert und sollte nur in Einzelfällen erwogen werden.

\section{Karzinome des Pankreaskorpus und -schwanzes}

Karzinome des Pankreaskorpus und -schwanzes werden häufig in einem sehr fortgeschrittenen Stadium diagnostiziert. Die 1- und 5-Jahres-Überlebensraten nach Linksresektion sind mit 50 versus $64 \%$ und 15 versus $17 \%$ tendenziell schlechter als nach Pankreaskopfresektionen [33]. In einer großen Studie an 590 Patienten mit Pankreaskorpus- und -schwanzkarzinom konnte durch Resektion nur bei Tumoren kleiner $4 \mathrm{~cm}$, ohne Lymphknotenbefall und ohne Fernmetastasen ein Überlebensvorteil im Vergleich zu Bypassverfahren erreicht werden [34].

Shimada et al. [35] publizierten eine Serie von 88 Patienten mit Karzinomen des Pankreaskorpus und -schwanzes, welche mit ausgedehnter Lymphadenektomie reseziert wurden. Die 1-, 3- und 5-Jahres-Überlebensraten betrugen in dieser Arbeit 76, 40 und 19\%. Unabhängige prognostische Faktoren waren ein Tumorbefall der Lymphknoten und der histologische Nachweis einer venösen Gefäßinfiltration. In einer aktuellen Studie von Kanda et al. [36] an 51 Patienten mit Pankreaskorpus- und -schwanzkarzinomen erwies sich in der multivariaten Analyse die Infiltration der A. lienalis, jedoch nicht die Infiltration der V. lienalis, als unabhängiger prognostischer Faktor.

Möglicherweise kann durch eine aggressivere Chirurgie die Resektionsrate und das Langzeitüberleben gesteigert werden. Die Pankreaslinksresektion mit En-bloc-Resektion des Truncus coeliacus wurde zum ersten Mal in Japan beschrieben [37]. Die Arbeitsgruppe von J.L. Cameron [38] beschrieb 2005 eine Kasuistik mit erfolgreicher Resektion von Pankreaskorpus und -schwanz, Milz, Magen und Truncus coeliacus. Hirano et al. [39] publizierten bei 23 Patienten mit En-bloc-Resektion des Truncus coeliacus eine R0-Resektionsrate von $91 \%$, eine OP-Mortalität von $0 \%$ und eine 5-Jahres-Überlebensrate von $42 \%$. Die Arbeitsgruppe konnte auch zeigen, dass durch die Mitresektion des Plexus coeliacus eine bessere Schmerzkontrolle erreicht werden konnte.

\section{Konklusion}

Durch die En-bloc-Resektion des Truncus coeliacus und der A. hepatica communis kann bei den meist fortgeschrittenen Pankreaskorpus- und -schwanzkarzinomen eine höhere R0Resektion erreicht werden. Ob mit diesen erweiterten Operationsverfahren das Langzeitüberleben verbessert werden kann, muss in zukünftigen Studien untersucht werden.

\section{Disclosure}

The authors declared no conflict of interest. 
1 Wagner M, Redaelli C, Lietz M, Seiler CA, Friess $\mathrm{H}$, Büchler MW: Curative resection is the single most important factor determining outcome in patients with pancreatic adenocarcinoma. Br J Surg 2004;91:586-594.

2 Adler G, Seufferlein T, Bischoff SC, Brambs H-J, Feuerbach S, Grabenbauer G, Hahn S, Heinemann V, Hohenberger W, Langrehr JM, Lutz MP, Micke O, Neuhaus H, Neuhaus P, Oettle H, Schlag PM, Schmid R, Schmiegel W, Schlottmann K, Werner J, Wiedenmann B, Kopp I: S3-Leitlinie «Exokrines Pankreaskarzinom» 2006 - Ergebnis einer «Evidenz»basierten Konsensuskonferenz (13.14.10.2006). AWMF online 2006 www.uni-duesseldorf de/WWW/AWMF/ll/032-010 pdf 2006.

3 Cameron JL, Riall TS, Coleman J, Belcher KA One thousand consecutive pancreaticoduodenectomies. Ann Surg 2006;244:10-15.

4 Pedrazzoli S, Beger HG, Obertop H, AndrénSandberg A, Fernandez-Cruz L, Henne-Bruns D, Luttges J, Neoptolemos JP: A surgical and pathological based classification of resective treatment of pancreatic cancer. Summary of an international workshop on surgical procedures in pancreatic cancer. Dig Surg 1999;16:337-345.

5 Farnell MB, Pearson RK, Sarr MG, DiMagno EP, Burgart LJ, Dahl TR, Foster N, Sargent DJ: A prospective randomized trial comparing standard pancreatoduodenectomy with pancreatoduodenectomy with extended lymphadenectomy in resectable pancreatic head adenocarcinoma. Surgery 2005 ; 138:618-628.

6 Henne-Bruns D, Vogel I, Luttges J, Kloppel G, Kremer B: Surgery for ductal adenocarcinoma of the pancreatic head: staging, complications, and survival after regional versus extended lymphadenectomy. World J Surg 2000;24:595-601.

7 Pedrazzoli S, DiCarlo V, Dionigi R, Mosca F, Pederzoli P, Pasquali C, Kloppel G, Dhaene K, Michelassi F: Standard versus extended lymphadenectomy associated with pancreatoduodenectomy in the surgical treatment of adenocarcinoma of the head of the pancreas: a multicenter, prospective, randomized study. Lymphadenectomy Study Group. Ann Surg 1998;228:508-517.

8 Yeo CJ, Cameron JL, Lillemoe KD, Sohn TA, Campbell KA, Sauter PK, Coleman J, Abrams RA, Hruban RH: Pancreaticoduodenectomy with or without distal gastrectomy and extended retroperitoneal lymphadenectomy for periampullary adenocarcinoma, part 2: randomized controlled trial evaluating survival, morbidity, and mortality. Ann Surg 2002;236:355-366.

9 Michalski CW, Kleeff J, Wente MN, Diener MK, Büchler MW, Friess H: Systematic review and meta-analysis of standard and extended lymphadenectomy in pancreaticoduodenectomy for pancreatic cancer. Br J Surg 2007;94:265-273.

10 Sobin LH, Gospodarowicz MK, Wittekind C: TNM Classification of Malignant Tumours (UICC International Union Against Cancer), 7th ed. New York, John Wiley and Sons, 2009.

11 Klimpfinger M, Hermanek P, Büchler MW, Neuhaus P, Wittekind C: Japanische Klassifikation maligner Pankreastumoren; Klassifikation maligner Tumoren des Gastrointestinaltrakts. Berlin, Springer, 2007 pp 295-306.

12 Kim YC, Park MS, Cha SW, Chung YE, Lim JS, Kim KS, Kim MJ, Kim KW: Comparison of CT and MRI for presurgical characterization of paraaortic lymph nodes in patients with pancreatico-biliary carcinoma. World J Gastroenterol 2008;14:2208-2212.
13 Lee MJ, Yun MJ, Park MS, Cha SH, Kim MJ, Lee JD, Kim KW: Paraaortic lymph node metastasis in patients with intra-abdominal malignancies: CT vs PET. World J Gastroenterol 2009;15:4434-4438.

14 Helmstaedter L, Riemann JF: Pancreatic cancer EUS and early diagnosis. Langenbecks Arch Surg 2008;393:923-927.

15 Soriano A, Castells A, Ayuso C, Ayuso JR, de Caralt MT, Gines MA, Real MI, Gilabert R, Quinto L, Trilla A, Feu F, Montanya X, Fernandez-Cruz L, Navarro S: Preoperative staging and tumor resectability assessment of pancreatic cancer: prospective study comparing endoscopic ultrasonography, helical computed tomography, magnetic resonance imaging, and angiography. Am J Gastroenterol 2004; 99:492-501.

16 Nakata B, Nishino H, Ogawa Y, Yokomatsu H, Kawasaki F, Kosaka K, Wada T, Suto R, Montani A, Hirakawa K: Prognostic predictive value of endoscopic ultrasound findings for invasive ductal carcinomas of pancreatic head. Pancreas 2005;30: 200-205.

17 Roche CJ, Hughes ML, Garvey CJ, Campbell F, White DA, Jones L, Neoptolemos JP: CT and pathologic assessment of prospective nodal staging in patients with ductal adenocarcinoma of the head of the pancreas. AJR Am J Roentgenol 2003;180: 475-480.

18 Berger AC, Watson JC, Ross EA, Hoffman JP: The metastatic/examined lymph node ratio is an important prognostic factor after pancreaticoduodenectomy for pancreatic adenocarcinoma. Am Surg 2004;70:235-240.

19 House MG, Gonen M, Jarnagin WR, D'Angelica M, DeMatteo RP, Fong Y, Brennan MF, Allen PJ: Prognostic significance of pathologic nodal status in patients with resected pancreatic cancer. J Gastrointest Surg 2007;11:1549-1555.

20 Pawlik TM, Gleisner AL, Cameron JL, Winter JM, Assumpcao L, Lillemoe KD, Wolfgang C, Hruban RH, Schulick RD, Yeo CJ, Choti MA: Prognostic relevance of lymph node ratio following pancreaticoduodenectomy for pancreatic cancer. Surgery 2007:141:610-618.

21 Riediger H, Keck T, Wellner U, zur Hausen A, Adam U, Hopt UT, Makowiec F: The lymph node ratio is the strongest prognostic factor after resection of pancreatic cancer. J Gastrointest Surg 2009; 13:1337-1344.

22 Sierzega M, Popiela T, Kulig J, Nowak K: The ratio of metastatic/resected lymph nodes is an independent prognostic factor in patients with node-positive pancreatic head cancer. Pancreas 2006;33:240-245.

23 Connor S, Bosonnet L, Ghaneh P, Alexakis N, Hartley M, Campbell F, Sutton R, Neoptolemos JP: Survival of patients with periampullary carcinoma is predicted by lymph node 8 a but not by lymph node $16 \mathrm{~b} 1$ status. Br J Surg 2004;91: 1592-1599.

24 Kayahara M, Nagakawa T, Ohta T, Kitagawa H, Ueno K, Tajima H, Elnemr A, Miwa K: Analysis of paraaortic lymph node involvement in pancreatic carcinoma: a significant indication for surgery? Cancer 1999;85:583-590.

25 Nakao A, Takeda S, Inoue S, Nomoto S, Kanazumi $\mathrm{N}$, Sugimoto H, Fujii T: Indications and techniques of extended resection for pancreatic cancer. World J Surg 2006;30:976-982.
26 Yamada S, Nakao A, Fujii T, Sugimoto H, Kanazumi N, Nomoto S, Kodera Y, Takeda S: Pancreatic cancer with paraaortic lymph node metastasis: a contraindication for radical surgery? Pancreas 2009;38:e13-e17.

27 Sakai M, Nakao A, Kaneko T, Takeda S, Inoue S, Kodera Y, Nomoto S, Kanazumi N, Sugimoto H Para-aortic lymph node metastasis in carcinoma of the head of the pancreas. Surgery 2005;137: 606-611.

28 Yekebas EF, Bogoevski D, Cataldegirmen G Kunze C, Marx A, Vashist YK, Schurr PG, Liebl L, Thieltges S, Gawad KA, Schneider C, Izbicki JR: En bloc vascular resection for locally advanced pancreatic malignancies infiltrating major blood vessels: perioperative outcome and long-term survival in 136 patients. Ann Surg 2008;247:300-309.

29 Riediger H, Makowiec F, Fischer E, Adam U, Hopt UT: Postoperative morbidity and long-term survival after pancreaticoduodenectomy with superior mesenterico-portal vein resection. J Gastrointest Surg 2006;10:1106-1115.

30 Kaneoka Y, Yamaguchi A, Isogai M: Portal or superior mesenteric vein resection for pancreatic head adenocarcinoma: prognostic value of the length of venous resection. Surgery 2009;145:417-425.

31 Amano H, Miura F, Toyota N, Wada K, Katoh K, Hayano K, Kadowaki S, Shibuya M, Maeno S, Eguchi T, Takada T, Asano T: Is pancreatectomy with arterial reconstruction a safe and useful procedure for locally advanced pancreatic cancer? J Hepatobiliary Pancreat Surg 2009;16:850-857.

32 Settmacher U, Langrehr JM, Husmann I, Eisele R, Bahra M, Heise M, Neuhaus P: Viszeralarterienrekonstruktionen mit Homografts bei Resektionen des Pankreas. Chirurg 2004;75:1199-1206.

33 Sohn TA, Yeo CJ, Cameron JL, Koniaris L, Kaushal S, Abrams RA, Sauter PK, Coleman J, Hruban RH, Lillemoe KD: Resected adenocarcinoma of the pancreas - 616 patients: results, outcomes, and prognostic indicators. J Gastrointest Surg 2000;4:567-579.

34 Fabre JM, Houry S, Manderscheid JC, Huguier M, Baumel H: Surgery for left-sided pancreatic cancer. Br J Surg 1996;83:1065-1070.

35 Shimada K, Sakamoto Y, Sano T, Kosuge T: Prognostic factors after distal pancreatectomy with extended lymphadenectomy for invasive pancreatic adenocarcinoma of the body and tail. Surgery 2006; 139:288-295.

36 Kanda M, Fujii T, Sahin TT, Kanzaki A, Nagai S, Yamada S, Sugimoto H, Nomoto S, Takeda S, Kodera Y, Morita S, Nakao A: Invasion of the splenic artery is a crucial prognostic factor in carcinoma of body and tail of the pancreas. Ann Surg 2010;251:483-487.

37 Mayumi T, Nimura Y, Kamiya J, Kondo S, Nagino M, Kanai M, Miyachi M, Hamaguchi K, Hayakawa $\mathrm{N}$ : Distal pancreatectomy with en bloc resection of the celiac artery for carcinoma of the body and tail of the pancreas. Int J Pancreatol 1997;22:15-21.

38 Makary MA, Fishman EK, Cameron JL: Resection of the celiac axis for invasive pancreatic cancer. J Gastrointest Surg 2005;9:503-507.

39 Hirano S, Kondo S, Hara T, Ambo Y, Tanaka E, Shichinohe T, Suzuki O, Hazama K: Distal pancreatectomy with en bloc celiac axis resection for locally advanced pancreatic body cancer: long-term results. Ann Surg 2007;246:46-51. 